(Smith; H. Kern.). - Krain: Urata-Thal am Triglav (Kră̌an; H. Freyn); Alpe "Koziek bei Höfflein" (Rastern; H. T. Z.); ohne nähere Fundortsangabe (Deschmann: H. Berl.). - Istrien, Görz etc.: Insel Isola (L oser; H. M. Pr.). - Görz (Krašan: H. Kern.). - Canale (lg. ?; H. Hofm.). - Kärnthen: Malborgeth (Ressmann; H. Pach.)

Habituell ähnelt $E$. Kerneri am meisten der E. Rostkoviana, von der sie aber leicht an dem vollständigen Mangel der drüsigen Behaarung zu unterscheiden ist. In diesem Merkmale stimmt sie mit $\boldsymbol{E}$. picta und $\boldsymbol{E}$. versicolor überein, die aber durch die breiten stumpfzähnigen Stengelblätter einerseits ( $E$. picta), durch die niederen weniger verzweigten Stengel, weniger zähnigen Blätter andererseits (E. versicolor) von $\boldsymbol{E}$. Kerneri sich unterscheiden. Die zwei letzterwähnten Arten stehen zu $\boldsymbol{E}$. Kerneri zweifellos in genetischen Beziehungen, die noch ihre Erörterungen finden werden.

Bei Bestimmung von Herbarexemplaren ist es oft von Nutzen, auf die braune Färbung, welche gerade die Blätter dieser Art häufig beim Trocknen annehmen, zu achten.

(Fortsetzung folgt.)

\title{
Weiden und Weidenbastarde
}

aus der

\section{Umgebung von Hohenstadt in Mähren.}

\author{
Von J. Panek, Bürgerschuldirector (Hohenstadt).
}

Angeregt durch die ausgezeichnete Arbeit A. v. Kerner's über "Niederösterreichische Weiden" im X. Bande der Verhandlungen der k. k. zoolog.-botanisch. Gesellschaft in Wien, beschäftigt sich der Verfasser seit vielen Jahren mit dem Studium der Weiden aus der nächsten Umgebung Hohenstadts.

Die Fundorte der nachstehend verzeichneten Weiden und Weidenbastarde sind einestheils Eisenbahnausstiche, anderntheils die sandigen Ufer der mährischen Sazawa und jene niedrigen Ausläufer des Gesenkes, die bei Hohenstadt das obere Marchbecken westlich begrenzen.

Das Terrain, auf dem die Weiden gesammelt wurden, hat eine Seehöbe von 290-350m; die mittlere Eintfernung von den höehsten Gipfeln des Gesenkes beträgt ungefähr $40 \mathrm{~km}$.

Einzelne schwierigere Bastarde hat der ansgezeichnete Salicologe Herr Hofrath A. Ritter v. Kerner gütigst determinirt, den grössten Theil des Weidenmateriales jedoch Herr Dr. C. Fritsch, Adjunct am botanischen Museum der $k$. k. Universität in Wien revidirt. Beiden Herren dankt der Verfasser hiemit für die ihm bewiesene Freundlichkeit. 


\section{A. Stammarten.}

Salix triandra Linn. $\alpha$. concolor Wm. et Grab. In der Umgebung häufig; $\beta$. discolor, seltener.

- alba Linn. Eine der am bäufigsten vorkommenden Weiden.

- fragilis Linn. Sebr häufig.

- purpurea Linn. Folgende Formen wurden beobachtet (Wimm. Salic. Europ. pag. 31-32):

eriantha. Kätzchen gross, $\mathbf{9}$ Kätzchen zur Zeit der Fruchtreife bis $8 \mathrm{~cm}$ lang. Im Sammelgebiete häufig.

gracilis. Kätzchen klein, zur Zeit der Blüthe $3-4 \mathrm{~mm}$ breit, Blatter fast lineal, kurz zugespitzt, Aeste dünn. Im Sammelgebiete häufig.

Lambertiana. Die Blätter paarweise gegenständig, gross, meist breitlanzettlich kurzgestielt. Häufig.

styligera. Fruchtknoten zngespitzt, Griffel sehr kurz, aber kenntlich. Selten.

Monströse Formen :

Ein Strauch mit androgynen Kätzchen, die or und $\mathbf{Q}$ Blüthen in der mannigfachsten Weise angeordnet, und zwar der obere Theil des Kätzchens $\sigma$, der untere $\mathcal{Q}$ oder umgekehrt, ferner der mittlere Theil weiblich, die übrigen Theile des Kätzchens männlich.

Die Staubbeutel erscheinen theilweise in Fruchtknoten umgebildet. Bei einzelnen Kätzchen sind fast sämmtliche Staubbeutel umgebildet, bei anderen finden sich zahlreiche Uebergänge zwischen langgestieiten unvollkommenen und sitzenden vollkommen ausgebildeten Fruchtknoten. Diese Bildung wurde an zwei Sträuchern beobachtet.

Fast sämmtliche Fruchtknoten des Kätzehens sind bis zur Basis getheilt, die Theile schmal kegelförmig und bogig gekrümmt. Das Kätzchen eihält hiedurch ein eigenthümliches Aussehen, es erscheint bedeutend breiter, als bei der normalen Form und die Kätzchenschuppen werden ganz verdecht. Diese Bildung scheint bei S. purpurea sehr selten zu sein; im Sammelgebiete wurde 1 Strauch beobachtet.

- daphnoides Vill. Ziemlich häufig, jedoch nur angepflanzt; ausschliesslich männliche Bäume.

- viminalis Linn. Formen:

vulgaris Kern. Im Sammelgebiete nicht häufig, nur 1 männlicher und einige $Q$ Sträncher. Ein $Q$ Strauch dadurch bemerkenswerth, dass der Griffel sehr verlängert, nach oben verbreitert erscheint und die Narben tief zweispaltig sind. tenuifolia Kern. Blätter $15 \mathrm{mal}$ länger als breit. Kätzchen klein, $24-25 \mathrm{~mm}$ lang, $4-5 \mathrm{~mm}$ breit. Ein of Stranch. 
- silesiaca Willd. Der normalen Form nahestehend. Blätter später fast ganz kahl, Griffel kurz. Zwei 9 Sträucher. Das Vorkommen dieser Weide bei ungefähr $350 \mathrm{~m}$ Seehöhe ist bemerkenswerth.

- cinerea Linn. Im Sammelgebiete minder bäufig. Ein Strauch mit monströsen Blüthen ist sehr bemerkenswerth. Die Fruchtknoten sind gedoppelt, der gemeinsame Fruchtknotenstiel ist $6-8 \mathrm{~mm}$ lang, im obersten Theile gespalten und die abnormal geformten Fruchtlnoten (umgebildete Staubbeutel) tragend, diese selbst sind sehr schwach behaart und von gelblichgrüner Farbe. Das ganze Kätzchen erscheint zur Zeit der vollen Blüthe freudig gelbgrün und gewährt bei seiner ansehnlichen Grösse (Länge $35 \mathrm{~mm}$, Breite $18 \mathrm{~mm}$ ) einen eigenthümlichen Anblick.

- caprea Linn. In der Umgebung häufig. Von abnormalen Formen and monstrosen Bildungen wurden beobachtet:

Ein kräftiger $Q$ Stranch zeigt an den Fruchtknoten eine bemerkenswerthe Narbenbildung; selbe sind tief zweispaltig nach aufwärts auseinanderspreizend. Die Kätzchen sind aussergewöhnlich gross und haben zur Zeit der Blüthe eine Länge von $4.5 \mathrm{~cm}$, unmittelbar vor der Fruchtreife eine solche von $9 \mathrm{~cm}$.

Bei einem kräftigen $\approx$ Strauche entwickeln sich aus einer Knospe 3 Kätzchen auf einmal; das mittlere ist das grösste, die seitlichen sind kleiner. Jedes Kätzchen hat seine eigene Deckschuppe, überdies werden alle drei in der Knospenlage von einer einzigen grossen Deckschuppe verhüllt. Finzelne Knospen bergen blos 2 Kätzehen in derselben Weise. Dieselbe Bildung zeigt auch noch ein $\mathbf{Q}$ Strauch der S. silesiaca.

An einem Strauche erscheinen die Staubbeutel in mehr oder minder vollkommener Weise in Fruchtknoten umgebildet.

Ein $\%$ Strauch besitzt tief getheilte, gedoppelte Fruchtknoten.

- aurita Linn. In der typischen Form bäufiger als S. caprea.

Folgende Formen wurden beobachtet:

spathulata Wimm. Von niedrigem Wuchse, Blätter steif, klein. länglich lanzettlich, auf der Unterseite blangran filzig. Hie und da an sonnigen sandigen Orten.

uliginosa Wim. Aeste aufrecht, langgestreckt, Blätter gross bis $10 \mathrm{~cm}$ lang und $4 \mathrm{~cm}$ breit, verkehrt eiförmig, kurz zugespitzt in den Blattstiel langkeilig verschmälert. An fenchten schattigen Orten.

Ein or Strauch ist dadurch merkwürdig, dass die sehr zahlreichen Blättchen des Kätzchenstieles allmälig in die Kätzchenschuppen übergehen. Die Staubgefässe bleiben in der Entwicklung zurïck und die Kätzchen gelangen niemals zur vollen Blüthe. 
Ein 9 Strauch zeigt insoferne eine abnorme Bildung, als die Kätzchen sehr dichtblüthig und die Kätzchenschuppen langzottig sind. Die Fruchtknoten stehen so dicht an der Spindel, dass sie sich auch noch nach der Blüthezeit gegenseitig berühren.

An einem 9 Strauche wurden eigenthümliche Nebenblätter beobachtet; selbe sind länglich lanzettlich oder auch halbspiessförmig, $10-12 \mathrm{~mm}$ lang, $2-4 \mathrm{~mm}$ breit.

\section{B. Bastarde.}

Salix fragilis $\times$ alba Wimm. Häufig in der Form S. viridis Fries.

- fragilis $\times$ triandra Wimm. Auch dieser Bastand kommt in verschiedenen Formen ziemlich häufig vor.

- pentandra $\times$ fragilis Wimm. Ein nicht blühender Strauch, der der Blattform nach der $S$. pentandra näher stehen dürfte.

- viminalis $\times$ purpurea Kern. S. rubra Huds. Formen:

a) latifolia Wimm. Q Pflanzen sehr häufig, aber nur 1 männlicher Strauch.

b) angustifolia Tausch; selten; zwei $\%$ und ein o" Strauch.

- subviminalis $\times$ purpurea Kern. S. Forbiana Smith. Nicht selten, jedoch nur weibliche Pflanzen. Das seltene Vorkommen der $0^{\star}$ Pflanzen dieser beiden Bastarde verdient bemerkt zu werden.

- superpurpurea $\times$ caprea. In je einem $o^{*}$ und $\mathbf{Q}$ Strauche. Die Blätter zeigen nur in ibrer Jugend die Behaarung der $S$. caprea, werden aber bald auf der Unterseite ganz kahl. Der männliche Strauch dürfte der S. Mauternensis nahe stehen. Die Staubfäden sind bis zur Mitte verwachsen.

- subpurpourea $\times$ cinerea Wimm. - S. sordida Kerner. Ein $\sigma^{7}$ Strauch, wolcher der $S$. cinerea sehr nahe steht. Die Staubfäden sind im untersten Theile oder bis zum ersten Drittel verwachsen.

Fin Strauch dieses Bastardes zeigt eine Umbildung der Staubbeutel in Fruchtknoten.

- aurita $\times$ purpurea Wimm. In den Formen: $\beta$. cinerascens Wimm. S. auritoides Kern. Ein weiblicher Strauch.

- glaucescens Wimm. S. superpurpureo $>$ aurita. Ein $\$$ und 3 männliche Sträucher; ausserdem wurden noch mehrere Exemplare dieses Bastardes in Blättern beobachtet.

- purpurea $\times$ silesiaca Wimm. Dieser Bastard wurde bisher in einem einzigen O Strauche aufgefunden, der die Mittelform zwischen den beiden Stammeltern darstellen dürfte. Das Vorkommen dieses Bastardes ist mit Rücksicht auf seinen Standort $(290 \mathrm{~m})$ interessant.

- caprea Xviminalis Wimm. S. sericans Tausch, in der Form Smithiana Wimm. Ein $\$$ Strauch hochwächsig, beim Bahn- 
hofe in Heilendorf nächst Hohenstadt. Ein zweiter Strauch bisher blos in Blättern.

Salix caprea $\times$ cinerea Wimm. S. Reichardtii Kerner. Ein kräftiger O Strauch.

- aurita $\times$ caprea Wimm. Bisher wurden 3 O Sträucher. dieses Bastardes aufgefunden, von denen der eine mehr zu S. caprea, der andere zu S. aurita hinneigt, während der dritte die Mittelform darstellen dürfte.

- aurita $\times$ cinerea Wimm. S. lutescens Kern. Zwei O Sträucher, von denen der eine gedoppelte Fruchtknoten besitzt. Die Kätzchen erscheinen infolge dessen sehr dichtblüthig. Eine merkwürdige Bildung zeigt ein ơ Stranch. Die Blüthen sind scheinbar 3 männig, da oberhalb einer Kätzchenschuppe 3 Staubgefässe ihren Ursprung haben. Dies trifft jedoch nur bei einzelnen Blüthen desselben Kätzchens zu, bei anderen erscheint nämlich der eine der beiden Staubfäden im unteren Theile oder höher oben gabelig gespalten, jeder Theil trägt einen Staubbeutel.

- caprea $\times$ silesiaca Wimm. Ein kräftiger $\subseteq$ Strauch. Die Fruchtknoten sind fast kahl, die Blätter breit eiförmig oder breit elliptisch, oberhalb dunkelgrün glänzend. Die Behaarung der Unterseite der Blätter jener der $S$. caprea sehr ähnlich, doch minder dicht und bläulichgrün.

- aurita $\times$ silesiaca Wimm. Dieser Bastard wurde in mehreren Q Sträuchern aufgefunden, von denen einige mehr zu S. aurita, die anderen wieder mehr zu $S$. silesiaca hinneigen. Form und Behaarung der Blätter ist bei jedem Strauche eigenartig. Die Fruchtknoten sind zumeist ganz kahl oder seltener im unteren Theile behaart. Formen mit vollständig behaarten Fruchtknoten wurden nicht aufgefunden. Wimmer unterscheidet in seinen Salic. Europ. neun Formen dieses Bastardes; nach der Beschreibung war es unmöglich, die hier aufgefundenen einzureihen. Das Vorkommen dieser hybriden Weide und der anderen Bastarde mit $S$. silesiaca muss mit Rücksicht auf die niedrige Lage des Fundortes und den Umstand, dass das eigentliche Vegetationsgebiet der $S$. silesiaca wenigstens $30 \mathrm{~km}$ entfernt liegt, als merkwürdig bezeichnet werden.

Ausser den angeführten wurden noch melrere andere Bastarde aufgefunden, jedoch bisher nur in Blättern, so dass deren Bestimmung und Bekanntmachung einem späteren Zeitpunkte vorbehalten bleiben muss. 